\title{
Modifiable Walking Pattern Generation using Real-Time ZMP Manipulation for Humanoid Robots
}

\author{
Bum-Joo Lee ${ }^{1}$, Daniel Stonier ${ }^{2}$, Yong-Duk Kim ${ }^{1}$, Jeong-Ki Yoo ${ }^{1}$ and Jong-Hwan Kim ${ }^{1}$, Senior Member, IEEE
}

\begin{abstract}
Complex navigational commands require a walking pattern generator that is able to modify the pattern at any point in the walking gait. This paper utilizes the 3D-LIPM(Linear Inverted Pendulum Model) for generating a walking pattern, but introduces a method that allows for manipulation of the ZMP over the convex hull of the foot polygon whilst in single support phase. This permits a range of dynamic walking states that are not achievable using the conventional 3D-LIPM. These walking states are defined as a feasible region. A real-time algorithm is then developed, which follows the cue from a complex navigational command exactly when the desired walking state is in the feasible region and chooses the nearest feasible motion when it lies outside the feasible region. The proposed scheme is both simulated and implemented on the humanoid robot HanSaRam-VII developed at RIT laboratory, KAIST.
\end{abstract}

\section{INTRODUCTION}

Many of the existing methods for humanoid walking control are constrained by the need to regulate foot positions without any consideration for navigation of the humanoid within a complex environment [1], [2]. To achieve more robust motion control in these environments, the robot must be able to adjust its walking state (center of mass motion) and step length to accommodate whatever path planning approach is used.

In most inverted pendulum based approaches which do not utilize ZMP manipulation rely on only landing-position adjustment control to change step length. These do not take the environment itself into consideration and have difficulty in catering for special conditions such as those in the stepping-stone problem. ZMP based approaches, however, can address these issues as they use a ZMP trajectory as the specified control input. The ZMP following approach can be achieved by inverse FFT or numerical calculation of the ZMP trajectory in the time domain [3], [4]. These two approaches are batch process methods. To generate a walking pattern they must calculate entire trajectories or piecewise trajectories over some time interval.

To solve this batch problem Kajita et al. introduced a preview control scheme using a cart-table model [5]. They successfully generated walking patterns that followed a given

\footnotetext{
${ }^{1}$ Department of Electrical Engineering and Computer Science, Korea Advanced Institute of Science and Technology (KAIST), 373-1, GuseongDong, Yuseong-Gu, Daejeon, 305-701, Republic of Korea (e-mail: $\{$ bjlee, ydkim, jkyoo, johkim\} @ rit.kaist.ac.kr). ${ }^{2}$ Yujin robot, Namsung-Plaza, 34530 Gasan-dong, Guemcheon-gu, Seoul, 153-802, Repu blic of Korea (e-mail: d.stonier@gmail.com).
}

reference ZMP trajectory. This method is a mixture of the ZMP based and the inverted pendulum based approaches.

The proposed method in this paper extends the conventional 3D-LIPM in such a way so that feasibility of desired motions is considered. By manipulating the ZMP with closed form functions, a simple and direct means to control the humanoid dynamics is realized. It is similar with the method proposed by Sugihara [6] in the usage of analytical solution of simplified equation of motion. Proposed method, however, enables control of $\mathrm{CM}$ velocity and step lengths directly and independently. This allows the humanoid to robustly plan for complex navigational command strategies, such as that needed for the stepping stone problem. Additionally, it provides feasible regions that are utilized to determine whether or not a desired navigational command is feasible. This was not achievable with a conventional 3D-LIPM control algorithm. It also removes the need for preview data such as that employed by the preview control scheme.

This paper is organized as follows. In Section II, the dynamics of the 3D-LIPM is reviewed and the subsequent equations of motion using the ZMP functions as input are derived. Section III develops the walking control algorithm. Section IV details the ZMP manipulation scheme and Section $\mathrm{V}$ presents experimental results on the humanoid robot HanSaRam-VII. Conclusions are provided in Section VI.

\section{Mathematical Model}

\section{A. Dynamics of 3D-LIPM}

When a humanoid robot is in single support phase, its dominant dynamics can be simply modeled as a single inverted pendulum (Fig. 1). The first practical implementation of this approach was done by Kajita et al. [7]. This method has a few significant practical advantages, namely:

- The dominant dynamics with simple equations.

- The decoupled equations of motion for the sagittal and lateral planes by assuming there is no vertical motion of the center of mass $\left(z=Z_{C}\right)$.

Fig. 1 illustrates the planar constraint for the inverted pendulum model. In this model, the dynamics is further simplified by assuming the leg is a weightless telescopic limb and the mass is concentrated at a single point as shown. With the assumption that $z=Z_{C}$, the dynamic equations are given as follows:

$$
\left[\begin{array}{l}
\ddot{y}-\frac{g}{Z_{C}} y \\
\ddot{x}-\frac{g}{Z_{C}} x
\end{array}\right]=-\frac{g}{Z_{C}}\left[\begin{array}{l}
y_{z m p} \\
x_{z m p}
\end{array}\right]
$$




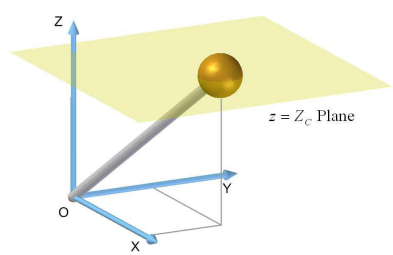

Fig. 1. 3D Linear Inverted Pendulum Model.

These equations provide the relationship between ZMP and center of mass (CM) motions. Refer to [6] for more detailed derivation.

\section{B. Equations of Motion}

From (1) the equation of motion for the 3D-LIPM in the sagittal plane with arbitrary ZMP function $p(t)$ is given by

$$
\ddot{x}-\frac{g}{Z_{C}} x=-\frac{g}{Z_{C}} p .
$$

Taking the Laplace transform of (2) and solving algebraically for $X(s)$,

$$
X(s)=\frac{s x(0)+\dot{x}(0)}{s^{2}-\left(1 / T_{C}\right)^{2}}-\frac{1}{T_{C}} \frac{1 / T_{C}}{s^{2}-\left(1 / T_{C}\right)^{2}} P(s),
$$

where $T_{C}=\sqrt{Z_{C} / g}$. Here the right hand side of (3) is composed of two parts: the first term represents the transform of the homogeneous solution, $X_{h}(s)$, and the second the transform of the particular solution, $X_{p}(s)$.

Solving for the homogeneous solution with the inverse Laplace Transform,

$$
x_{h}(t)=\cosh \left(t / T_{C}\right) x(0)+\sinh \left(t / T_{C}\right) v(0) T_{C} .
$$

where $v(0)$ is $\dot{x}(0)$. Similarly, utilizing the convolution property for Laplace transforms:

$$
x_{p}(t)=-\frac{1}{T_{C}} \int_{0}^{t} \sinh \left(\tau / T_{C}\right) p(t-\tau) d \tau .
$$

Similarly,

$$
\begin{aligned}
T_{C} v(t) & =\sinh \left(t / T_{C}\right) x(0)+\cosh \left(t / T_{C}\right) v(0) T_{C} \\
& -\frac{1}{T_{C}} \int_{0}^{t} \cosh \left(\tau / T_{C}\right) p(t-\tau) d \tau .
\end{aligned}
$$

The general solutions of (1) are represented in state space form at $t=T$ as follows:

$$
\left[\begin{array}{c}
x_{f} \\
T_{C} v_{f}
\end{array}\right]=\left[\begin{array}{cc}
C_{T} & S_{T} \\
S_{T} & C_{T}
\end{array}\right]\left[\begin{array}{c}
x_{i} \\
T_{C} v_{i}
\end{array}\right]-\frac{1}{T_{C}}\left[\begin{array}{l}
\int_{0}^{T} S_{t} \bar{p}(t) d t \\
\int_{0}^{T} C_{t} \bar{p}(t) d t
\end{array}\right]
$$

where $\left(x_{i}, v_{i}\right) /\left(x_{f}, v_{f}\right)$ represents initial/final position and velocity of the CM in the sagittal. $S_{t}$ and $C_{t}$ are defined as $\cosh \left(t / T_{C}\right)$ and $\sinh \left(t / T_{C}\right)$ with time constant $T_{C}=$ $\sqrt{Z_{C} / g}$. The functions $p(t)$ is ZMP trajectory for the sagittal. Lastly, $\bar{p}(t)=p(T-t)$. Solutions for motion in the lateral plane may be similarly obtained.

The first terms in the right hand side of (7) indicate homogeneous (non-forced) solutions. The latter term represents additional states (particular or forced solutions). These allow more extensive and unrestricted motions by manipulating ZMP trajectories. In the conventional 3D-LIPM technique by Kajita, only the homogenous part of the solution has been considered. While in single support phase this technique assumes the ZMP is fixed at the point of contact. Subsequently, the dynamics for the CM motion (1) is pre-determined and un-modifiable throughout single support phase.

This has the undesirable effect that CM motion cannot be flexibly adjusted in response to dynamically changing navigational commands while in single support phase. This limits the performance of the humanoid - it cannot accelerate or decelerate, nor can it vary its step length or walking period in the same manner that humans are easily able to achieve. Consequently, the model constraint requiring the ZMP to be fixed at the point of contact needs to be relaxed in order to achieve some level of control over the CM motions.

\section{ZMP Stable Region}

Huang introduced the concept of a desired stable region over which the ZMP may be safely varied [8]. This is illustrated in Fig. 2. $P_{\min }, P_{\max }, Q_{\min }$ and $Q_{\max }$ define the allowable stable region over which the ZMP may vary in $\mathrm{x}$ and y directions. The objective of the next section is to devise an algorithm that can utilize the permissible variation in the ZMP as defined above to generate dynamically modifiable CM motions.

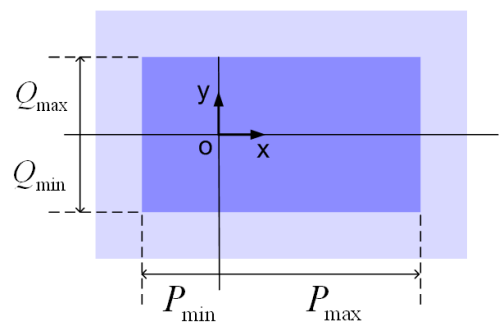

Fig. 2. ZMP stable region on foot. The outer region is the stability margin and the inner region is the desired stable region.

\section{Modifiable Walking Pattern}

\section{A. Command State}

This research focuses on generating a form of control that is able to perform complex navigational tasks such as stone stepping. This generalizes to obstacle avoidance for foot placement planning. To do this, the control requires a minimal command set that allows for configuration of a walking pattern based on the 3D-LIPM. The set of parameters necessary to derive a walking pattern based on the 3DLIPM includes state transition times and step lengths. This instructional set is defined as the command state.

Definition 1: CS (Command State) is defined as follows:

$$
\mathbf{c} \equiv\left[\begin{array}{lllll}
T_{s s} & T_{d s} & W_{L} & W_{F l} & W_{F r}
\end{array}\right]^{T},
$$

where

$T_{s s}:$ (commanded) single support time, 
$T_{d s} \quad$ : double support time,

$W_{L} \quad$ : step length of sagittal motion (both legs),

$W_{F l}$ : sidestep length of lateral motion (left leg) and

$W_{F r}$ : sidestep length of lateral motion (right leg).

Note that sidestep lengths in the lateral plane correspond to sideways movement.

\section{B. Walking State}

Since the inverted pendulum is modeled as a point mass, its state in $3 \mathrm{D}$ space can be simply represented in terms of position and linear velocity. Here, the CM position and velocity of the 3D-LIPM in both sagittal and lateral planes are defined as the WS of a two dimensional vector in each plane in the following.

Definition 2: WS (Walking State) is defined as follows:

$$
\begin{aligned}
& \mathbf{x}=\left[\begin{array}{ll}
x & T_{C} v
\end{array}\right]^{T} \text { for sagittal motion } \\
& \mathbf{y}=\left[\begin{array}{ll}
y & T_{C} w
\end{array}\right]^{T} \text { for lateral motion, }
\end{aligned}
$$

where $v$ and $w$ represent the velocity in each plane, respectively, and they are multiplied by the time constant, $T_{C}$.

\section{Feasible Region}

While in single support phase, the robot can vary the walking state by manipulating the ZMP trajectory. However, this does not mean that the robot has the ability to change its walking state to any desired one. This is due to both dynamic (ZMP boundary, surface friction, etc.) and kinematic (joint velocity and acceleration, joint angle range, etc.) constraints. Thus, the desired WS at the end of the single support phase must fall within some permissable and bounded region (Fig. 3 ). This region is defined as the Feasible Region, $F R$, and is detailed in Section IV.

Definition 3: Given the initial WS $\mathbf{w}_{i}, Z_{\min }, Z_{\max }$ and some $T_{\max } \geq 0$, a set is defined as the feasible region, $F R$, if $\forall \mathbf{w}_{f} \in F R$, there exists a ZMP function $z(t)$ with the following properties

such that

$$
\begin{gathered}
z:\left[0, T_{\max }\right] \rightarrow \mathbb{R}^{2}, \\
Z_{\min } \leq z(t) \leq Z_{\max }
\end{gathered}
$$

$$
f_{z}\left(\mathbf{w}_{i}\right)=\mathbf{w}_{f}
$$

where $f$ is the state transition function for either the sagittal or lateral plane (7). $F R^{c}$ is defined as the infeasible region.

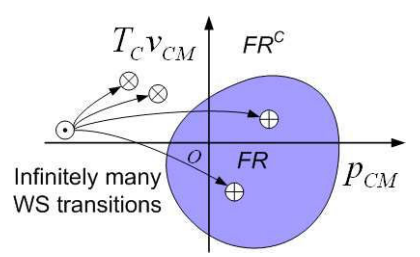

Fig. 3. Feasible and infeasible regions on WS plane $(\odot$ : initial WS, $\oplus$ : final WS $\in F R, \otimes$ : final WS $\in F R^{c}$ ).

\section{Desired Walking State}

Since walking is a repetitive motion, its behavior can be described by identifying the walking state at particular points in the motion in a similar fashion to the way Poincare Maps are used in dynamics. Any point in the walking pattern may be chosen for this purpose, however, in the analysis that follows, it is assumed that a point of interest (the walking state) is captured at the end of each single support phase. This provides two snapshots of the walking state within each cycle of the walking gait, one at the end of the left support phase and the other at the end of the right support phase. These adequately characterize the periodic nature of the walking pattern from one cycle to the next.

Now, when a robot follows a given CS exactly, it is assumed that

1) The robot's state is in steady state.

2) ZMP trajectories stay at the center of the ZMP stable margin.

These two conditions correspond directly with a walking pattern that represents steady state motion for the 3D-LIPM in which the particular solutions of (7) are zero (homogenous solutions only). In this situation, the walking state at the end of each single support phase will be identical. This state will be referred to as the desired walking state.

\section{E. Deriving Desired Walking State from Command State}

To derive the desired WS from the CS, it is enough to observe just one period of the walking configuration (steady state). One period of the robot's walking motion is illustrated in Fig. 4.

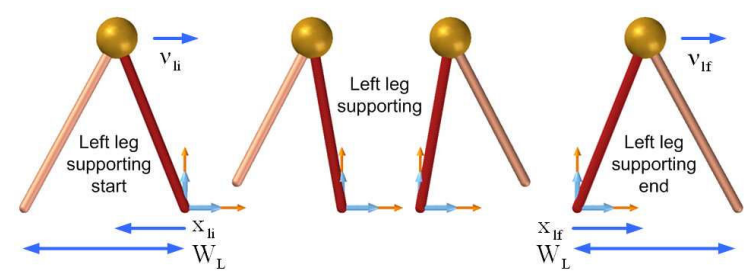

(a)
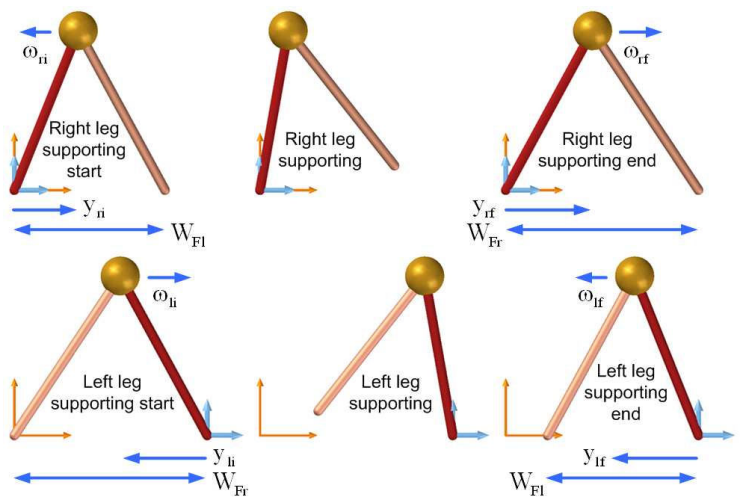

(b)

Fig. 4. Steady state motion. The darker leg indicates the supporting leg. (a) Sagittal plane. (b) Lateral plane. 
1) Sagittal Motion: Fig. 4 (a) illustrates sagittal motions. Motion begins with the left single support phase before switching to double support phase in which the CM travels with constant velocity for a pre-specified time $T_{d s}$. At this point the robot then switches to right single support phase. Since the sagittal motion is symmetrical each single support phase begins with the same configuration.

From the equation of sagittal motion (7) with $\bar{p}=0$, following two state equations are obtained during single support phase, which represent left and right single support phases, respectively:

$$
\mathbf{x}_{l f}=A \mathbf{x}_{l i}, \quad \mathbf{x}_{r f}=A \mathbf{x}_{r i},
$$

where

$$
A=\left[\begin{array}{ll}
C_{T_{s s}} & S_{T_{s s}} \\
S_{T_{s s}} & C_{T_{s s}}
\end{array}\right]
$$

During double support phase, the two equations of motion are given as follows:

$$
\mathbf{x}_{l i}=B \mathbf{x}_{r f}+C, \quad \mathbf{x}_{r i}=B \mathbf{x}_{l f}+C
$$

where

$$
B=\left[\begin{array}{cc}
1 & T_{d s} / T_{C} \\
0 & 1
\end{array}\right] \text { and } C=\left[\begin{array}{c}
-W_{L} \\
0
\end{array}\right] .
$$

As mentioned above, the velocities are controlled to be constant in double support phase. From (8) and (9), homogenous solutions for the sagittal steady state motion of the CM are given by

$$
\mathbf{x}_{l f}=\mathbf{x}_{r f}=(A B-I)^{-1} A C,
$$

The sagittal desired WS ${ }^{d} \mathbf{x}$ whilst in single support phase, becomes $\mathbf{x}_{l f}$ or $\mathbf{x}_{r f}$ for the left or right support phase, respectively.

2) Lateral Motion: By the same process, lateral steady state motion of the $\mathrm{CM}$ can be expressed by

$$
\begin{aligned}
\mathbf{y}_{l f} & =\left(I-(A B)^{2}\right)^{-1}(A B A D+A C), \\
\mathbf{y}_{r f} & =\left(I-(A B)^{2}\right)^{-1}(A B A C+A D),
\end{aligned}
$$

where

$$
\begin{aligned}
& A=\left[\begin{array}{ll}
C_{T_{s s}} & S_{T_{s s}} \\
S_{T_{s s}} & C_{T_{s s}}
\end{array}\right], B=\left[\begin{array}{cc}
1 & T_{d s} / T_{C} \\
0 & 1
\end{array}\right], \\
& C=\left[\begin{array}{c}
-W_{F r} \\
0
\end{array}\right] \text { and } D=\left[\begin{array}{c}
W_{F l} \\
0
\end{array}\right] .
\end{aligned}
$$

Again, the lateral desired WS ${ }^{d} \mathbf{y}$ whilst in single support phase becomes $\mathbf{y}_{l f}$ or $\mathbf{y}_{r f}$ for the left/right support phase, respectively.

\section{F. Target Walking State from Desired Walking State}

When the desired WS is in the $F R$, it is possible to move the current WS to the desired one. That is, the desired WS becomes the target WS for the control algorithm. However, when the desired WS is in $F R^{c}$ then the target WS is defined as the walking state on the boundary of $F R$ for which the norm of the error between target and desired WS is a minimum (Fig.5).

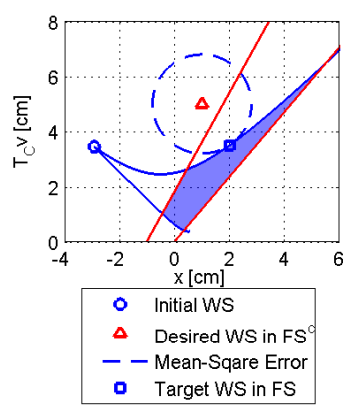

(a)

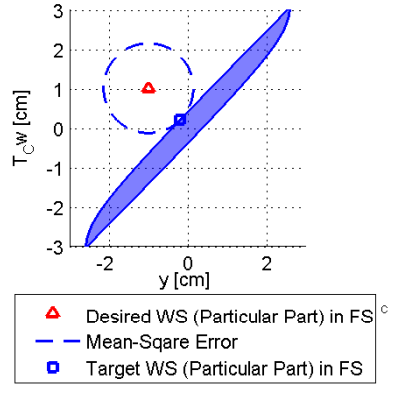

(b)
Fig. 5. Infeasible cases. (a) Sagittal plane. (b) Lateral plane (particular solution only).

\section{ZMP MANIPULATION}

\section{A. ZMP Manipulation Scheme}

In this section, the analytical method to control ZMP trajectory with closed form functions is introduced. Controlling the ZMP trajectory means controlling acceleration of $\mathrm{CM}$ and is effected the particular part of (7). The primary factors constraining the walking pattern in single support phase include the single support time and the ZMP trajectory functions in both sagittal and lateral planes. To formulate these concepts, let $T_{s t}$ represent the state transition time from the current WS to the final(desired) WS in the single support phase, and define the ZMP functions $p(t)$ and $q(t)$ for the sagittal and lateral planes on this interval. Consequently, given the current WS, $T_{s t}, p(t), q(t)$ represent the parameters that influence the WS at the end of the single support phase.

Note that there are infinitely many ZMP trajectories to transfer current WS to a desired WS. However, it is both essential and desirable that ZMP functions exhibit the following characteristics:

1) Closed form.

2) Minimum number of orders for real-time calculation.

3) Flow property: $z m p_{t}\left(z m p_{s}\left(z m p_{0}\right)\right)=z m p_{t+s}\left(z m p_{0}\right) \forall t, s \in\left[0, T_{s t}\right]$.

4) Maximum coverage of $F R$.

Among infinitely many candidate functions, it can be shown any arbitrary ZMP function can be equivalently represented by a simple step function. That is, the walking state obtained at the end of a single support phase given an arbitrary function can be similarly obtained from an appropriately selected step function. In other words, FR made by step function is maximum.

However, there remains a time coupling problem. The state transition time, $T_{s t}$, must also be determined along with the parameters for the step functions. Variation in the feasibility of motion is of primary importance in the sagittal direction (variation in walking speed, step length), while lateral motion is often restricted by kinematic constraints (caused by collision between the humanoid's legs). To reflect this, $T_{s t}$ will be appropriately configured from the sagittal equations of motion rather than the lateral equations.

To completely specify the ZMP trajectories in single support phase would require determination of five parameters 
(for step amplitudes and switching times for $p(t)$ and $q(t)$ and state transition time). In terms of implementation, it is desirable to reduce this by one so that the parameters may be exactly determined from the four equations of motion (7). Subsequently, the use of a constant function was investigated for the sagittal ZMP function (Fig. 6). Here $P$ and $Q$

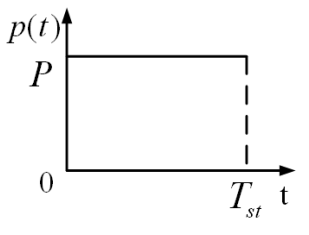

(a)

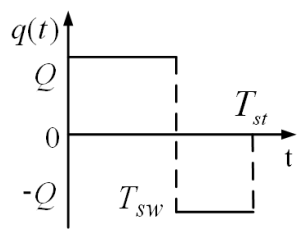

(b)
Fig. 6. Proposed ZMP functions. (a) Constant function (sagittal plane). (b) Step function (lateral plane).

are the constant and step function amplitudes, $T_{s w}$ is the switching time and $T_{s t}$ is the state transition time in the single support phase. This use of a constant function would also allow the state transition time to be determined from the desired walking state in the sagittal plane. A comparison of the feasible regions for a step and constant function is illustrated in Fig. 7, where the feasible regions are obtained by varying $T_{s t}$ and $P$. Here, $T_{s w}$ varies from 0 to $T_{s t}$ to induce maximum $F R$ in case of step function (a).

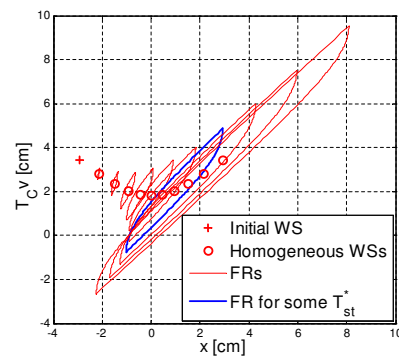

(a)

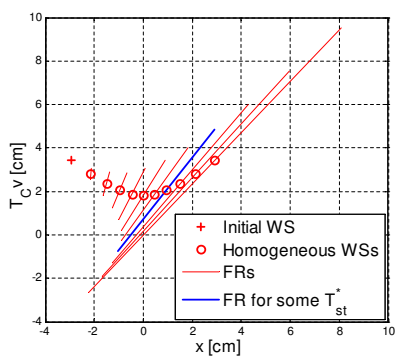

(b)
Fig. 7. Two feasible regions for the sagittal plane with respect to ZMP functions. ( $0 \leq T_{s t} \leq 0.4,-1 \leq P \leq 1$ ) (a) $F R$ with step function. (b) $F R$ with constant function.

Each graph displays a sequence of feasible sets with respect to a discrete set of time values. Keeping in mind that the $F R$ is the union of all these sets over continuous time. It can be seen that the effects of utilizing a constant function in preference to a step function does not reduce the feasibility of desired motions to a significant extent. Since this allows a more efficient implementation, this is deemed acceptable.

With these two ZMP functions ZMP trajectories can be manipulated on both planes to drive the robot's CM to the desired WS from the current WS. Note that this scheme can be adopted at any point in the single support phase in order to modify and adjust the robot's motion throughout this phase. This proposed scheme is very simple and covers almost all of the maximum usable $F R$ in both planes.

\section{B. Boundaries of Feasible Regions}

With the proposed two ZMP functions, the boundary of the FR must be investigated to judge whether the desired WS is in either $F R$ or $F R^{c}$.

1) Sagittal Motion: In the sagittal motion, a constant ZMP function is used to manipulate the ZMP trajectory. Thus there are two control parameters to manipulate WS: $T_{s t}$ and $P$. The boundary of $F R$ is determined by minimum and maximum values of $T_{s t}$ and $P$.

2) Lateral Motion: In the lateral plane with a step ZMP function, there remain two undetermined control parameters, $Q$ and $T_{s w}$. These are the step function amplitude and the switching time, respectively. Note that $T_{s t}$ is no longer a parameter as it is determined by the sagittal motion analysis. From (7), the homogeneous part is determined by the prespecified $T_{s t}$. Therefore, it is only necessary to calculate the particular solution. The Feasible Regions illustrated in Fig. 5 (colored regions) provide a criteria which classifies a desired WS as feasible or not.

\section{EXPERIMENTS}

Walking patterns were generated for the small-sized humanoid robot, HanSaRam-VII [9], [10]. Note that the proposed method is not limited to small-sized robots.

\section{A. Simulation Results}

Walking patterns for simulations were realized with following CS list:

- Initial $\mathbf{c}=\left[\begin{array}{llllll}0.4 & 0.2 & 6.0 & 6.0 & 6.0\end{array}\right]^{T}$.

- After $1^{\text {st }}$ step $\mathbf{c}=\left[\begin{array}{llllll}0.8 & 0.2 & 6.0 & 8.0 & 5.0\end{array}\right]^{T}$.

- After $5^{\text {th }}$ step $\mathbf{c}=\left[\begin{array}{lllll}0.5 & 0.2 & 4.0 & 5.0 & 6.0\end{array}\right]^{T}$.

- After $6^{\text {th }}$ step $\mathbf{c}=\left[\begin{array}{lllll}0.4 & 0.2 & -2.0 & 8.0 & 5.0\end{array}\right]^{T}$.

- After $7^{t h}$ step $\mathbf{c}=\left[\begin{array}{llllll}0.4 & 0.2 & -5.0 & 8.0 & 5.0\end{array}\right]^{T}$.

- After $8^{\text {th }}$ step $\mathbf{c}=\left[\begin{array}{lllll}0.4 & 0.2 & 0.0 & 6.0 & 6.0\end{array}\right]^{T}$.

where time units are given in seconds and length units in centimeters, respectively. Note that the each CS was not informed to the robot until the specified time to examine the effect of immediate CS variation.

Fig. 8 shows the generated walking pattern using the proposed scheme. As illustrated, the robot follows the CS

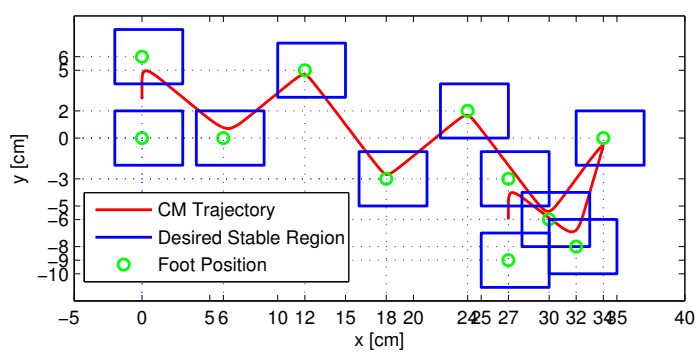

Fig. 8. Generated walking pattern using the proposed method. Desired stable region is set as $P_{\max }=3 \mathrm{~cm}, P_{\min }=-2 \mathrm{~cm}, Q_{\max }=2 \mathrm{~cm}, Q_{\min }=-$ $2 \mathrm{~cm}$.

exactly, even when the requested walking patterns varied suddenly, that is, the sudden side and backward walking motions near the end of the path. 


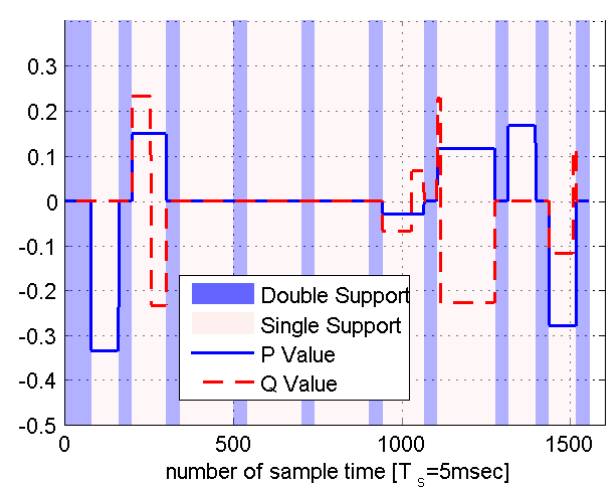

Fig. 9. Control parameters $\left(T, P, T_{s w}, Q\right)$

Fig. 9 illustrates changes in the ZMP trajectories (relative to the center of the supporting foot). ZMP variations can be seen to occur whenever the CS changes. This corresponds to forcing particular solutions that accelerate or decelerate the $\mathrm{CM}$ until the current walking state matches the desired walking state. When the walking pattern is steady-state, solutions are homogenous and the command state is followed using the conventional 3D-LIPM solution from step to step. Note that the maximum variation in the ZMP is less than $1 \mathrm{~cm}$ in either the sagittal or lateral planes. This is well within the limits imposed by the stable margin, even though dramatic variations in CS were commanded.

\section{B. Experimental Results}

Experimental conditions were exactly the same as those for the simulation. Footsteps successfully follow the designated pattern instructed by the command state (Fig. 10). Note that feed-back compensation was not used so that the proposed control algorithm could be analyzed solely on its own merits. Subsequently, there existed some noise and offset when compared to the simulation result. However, the ZMP trajectory was still within the desired ZMP stable region the robot was able to perform a stable walk. For more stable walking, momentum control could be adapted to consider full body effects.

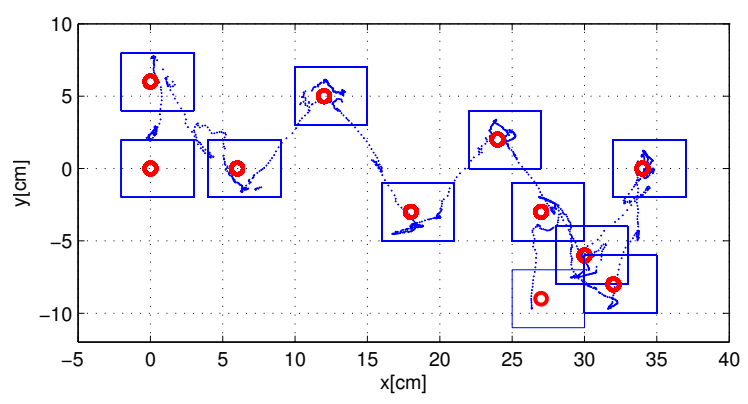

Fig. 10. ZMP trajectory on the ZMP stable margin. The circle and rectangle indicates the center of foot position and desired ZMP stable margin $\left(P_{\max }=3 \mathrm{~cm}, P_{\min }=-2 \mathrm{~cm}, Q_{\max }=2 \mathrm{~cm}, Q_{\min }=-2 \mathrm{~cm}\right)$, respectively. The ZMP trajectory is represented by the dotted line.

Fig. 11 shows a sequence of snap shots which capture the foot placements.. Refer to [11] for a movie clip.

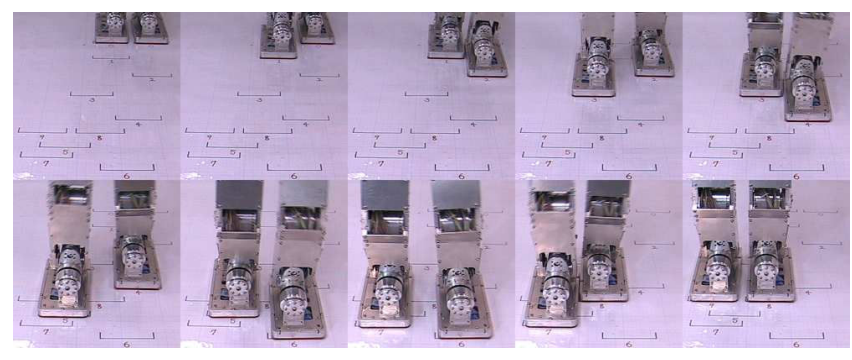

Fig. 11. Snap shots of experimental results (sequentially from left to right and top to bottom) [11].

\section{CONCLUSIONS}

A modifiable walking pattern generator was introduced and implemented on the humanoid robot HanSaRam-VII. The proposed method extended the conventional 3D-LIPM method by manipulating the ZMP while in single support phase. Using this, the humanoid robot could change both sagittal and lateral step length as well as the period of the walking pattern on command. The construction of feasible regions in both sagittal and lateral phase space was used as a criteria to determine the dynamic feasibility of desired motions.

\section{REFERENCES}

[1] P. Michel, J. Chestnutt, S. Kagami, K. Nishiwaki, J. Kuffner, and T. Kanade, "Online environment reconstruction for biped navigation" in Proc. of IEEE Int. Conf. on Robotics and Automations, Orlando, Florida, May 2006, pp. 3089-3094.

[2] J. Chestnutt, P. Michel, K. Nishiwaki, J. Kuffner, and S. Kagami, "An intelligent joystick for biped control" in Proc. of IEEE Int. Conf. on Robotics and Automations, Orlando, Florida, May 2006, pp. 860-865.

[3] H.-o. Lim, and A. Takanishi, "Realization of Continuous Biped Walking" in Proc. of IEEE Int. Conf. on Systems, Man and Cybernetics, Tucson, Arizona, Oct. 2001, pp. 1630-1635.

[4] K. Nishiwaki, S. Kagami, Y. Kuniyoshi, M. Inaba, and H. Inoue, "Online Generation of Humanoid Walking Motion based on a Fast Generation Method of Motion Pattern that Follows Desired ZMP" in Proc. of IEEE Int. Conf. on Intelligent Robots and Systems, Lausanne, Switzerland, Oct. 2002, pp. 2684-2689.

[5] S. Kajita, F. Kanehiro, K.Kaneko, K.Fujiwara, K.Harada, K.Yokoi, and H. Hirukawa, "Biped walking pattern generation by using preview control of zero-moment point" in Proc. of IEEE Int. Conf. on Robotics and Automations, Taipei, Taiwan, Sep 2003, pp. 1620-1626.

[6] T. Sugihara and Y. Nakamura, "A Fast Online Gait Planning with Boundary Condition Relaxation for Humanoid Robots" in Proc. of IEEE Int. Conf. on Robotics and Automations, Barcelona, Spain, Apr 2005, pp. 305-310.

[7] S. Kajita, F. Kanehiro, K. Kaneko, K. Yokoi, and H. Hirukawa, "The 3D Linear Inverted Pendul Mode: A simple modeling for a biped walking pattern generation" in Proc. of IEEE/RSJ Int. Conf. on Intelligent Robots and Systems, Maui, Hawaii, USA, Oct. 29 - Nov. 03, 2001, pp. 239-246.

[8] Q. Huang and Y.Nakamura, "Sensory Reflex Control for Humanoid Walking” IEEE Trans. on Robotics, vol. 21, Oct. 2005, pp. 977-984.

[9] J.-H. Kim, D.-H. Kim, Y.-J. Kim, K.-H. Park, J.-H. Park, C.-K. Moon, K. T. Seow, and K.-C. Koh, "Humanoid robot hansaram: Recent progress and development," J. of Advanced Computational Intelligence \& Intelligent Informatics, vol. 8, no. 1, pp. 45-55, Jan. 2004.

[10] Y.-D. Kim, B.-J. Lee, J.-K. Yoo, and J.-H. Kim, "Compensation for the Landing Impact Force of a Humanoid Robot by Time Domain Passivity Approach" in Proc. of IEEE Int. Conf. on Robotics and Automations, Orlando, Florida, May 2006, pp. 1225-1230.

[11] B.-J. Lee, D. Stonier, Y.-D. Kim, J.-K. Yoo, and J.H. Kim, "Modifiable Walking Pattern with HanSaRamVII" at http://rit.kaist.ac.kr/ ritlab/research/HanSaRam/ Modifiable_Walking_Pattern.wmv, Nov. 2006. 